\title{
The Effect of Dorsal Angulation on Distal Radioulnar Joint Arthrokinematics Measured Using Intercartilage Distance
}

\author{
Braden Gammon, MD, MSc, FRCSC ${ }^{1}$ Emily Lalone, $\mathrm{PhD}^{2}$ Masao Nishiwaki, MD, $\mathrm{PhD}^{3}$
} Ryan Willing, $\mathrm{PhD}^{2}$ James Johnson, $\mathrm{PhD}^{4}$ Graham J. W. King, MD, MSc ${ }^{5}$

\footnotetext{
${ }^{1}$ Division of Orthopedics, The Ottawa Hospital, University of Ottawa, Ottawa, Ontario, Canada

${ }^{2}$ Department of Mechanical and Materials Engineering, University of Western Ontario, London, Ontario, Canada

${ }^{3}$ Department of Orthopaedic Surgery, Kawasaki Municipal Hospital, Kawasaki-ku, Kawasaki, Japan

${ }^{4}$ Department of Mechanical and Materials Engineering, Lawson

Health Research Institute, London, Ontario, Canada

${ }^{5}$ Division of Orthopedic Surgery, Roth McFarlane Hand and Upper

Limb Centre, St. Joseph's Health Care, London, Ontario, Canada
}

\begin{abstract}
Address for correspondence Braden Gammon, MD, MSc, FRCSC, Division of Orthopedics, The Ottawa Hospital, University of Ottawa, Civic Campus (J159), 1053 Carling Avenue, Ottawa, ON K1Y 4E9, Canada (e-mail: bgammon@toh.on.ca).
\end{abstract}

J Wrist Surg 2019;8:10-17.

\begin{abstract}
Keywords

- arthrokinematics

- contact

- distal radioulnar joint

- intercartilage distance

- triangular fibrocartilage complex

Background The effects of dorsal angulation deformity on in vitro distal radioulnar joint (DRUJ) contact patterns are not well understood.

Purpose The purpose of this study was to utilize intercartilage distance to examine the effects of forearm rotation angle, distal radius deformity, and triangular fibrocartilage complex (TFCC) sectioning on DRUJ contact area and centroid position.

Methods An adjustable implant permitted the creation of simulated intact state and dorsal angulation deformities of 10,20 , and 30 degrees. Three-dimensional cartilage models of the distal radius and ulna were created using computed tomography data. Using optically tracked motion data, the relative position of the cartilage models was rendered and used to measure DRUJ cartilage contact mechanics.

Results DRUJ contact area was highest between 10 and 30 degrees of supination. TFCC sectioning caused a significant decrease in contact area with a mean reduction of $11 \pm 7 \mathrm{~mm}^{2}$ between the TFCC intact and sectioned conditions across all variables. The position of the contact centroid moved volarly and proximally with supination for all variables. Deformity had a significant effect on the location of the contact centroid along the volar-dorsal plane.

Conclusion Contact area in the DRUJ was maximal between 10 and 30 degrees of supination during the conditions tested. There was a significant effect of simulated TFCC rupture on contact area in the DRUJ, with a mean contact reduction of $11 \pm 7$ $\mathrm{mm}^{2}$ after sectioning. Increasing dorsal angulation caused the contact centroid to move progressively more volar in the sigmoid notch.
\end{abstract}

received

March 14, 2018

accepted

June 15, 2018

published online

August 15, 2018
Copyright $\odot 2019$ by Thieme Medical

Publishers, Inc., 333 Seventh Avenue, New York, NY 10001, USA

Tel: +1(212) 584-4662.
DOI https://doi.org/

10.1055/s-0038-1667303. ISSN 2163-3916. 
Distal radius fractures are the most common type of upper extremity fracture in the United States. ${ }^{1}$ Factors such as osteopenia, comminution, age over 60 years, and a high degree of initial displacement may predispose these to malunion. ${ }^{2}$ Residual dorsal angulation is the most common deformity, and the consequences of this have been the most widely studied. Specifically, residual dorsal angulation can alter forearm mechanics, with effects on both the range/axis of forearm rotation ${ }^{3-5}$ and torque required for prosupination. $^{6,7}$ Moreover, increased dorsal angulation may cause dorsal intercalated segmental instability ${ }^{8}$ and change the excursion and moment arms of the wrist muscles. ${ }^{9,10}$

The consequences of distal radius malunion on the distal radioulnar joint (DRUJ) have been the subject of further enquiry. Dorsal angulation of the distal radius has a significant effect on the DRUJ, causing incongruity, ${ }^{11}$ instability, ${ }^{12}$ and abnormal load transfer across the joint. ${ }^{13}$ Persistent disability from malunion has been observed clinically, with symptoms including ulnar-sided wrist pain, deformity, restricted forearm rotation, and limitations in grip strength. ${ }^{14,15}$ Dysfunction related to these may be exacerbated in the setting of associated triangular fibrocartilage complex (TFCC) rupture and DRUJ instability. ${ }^{16}$ These symptoms may, in part, relate to the biomechanical effects of distal radius malunion on the DRUJ.

Arthrokinematics, or the specific movement of joint surfaces, ${ }^{17}$ are not well understood for the DRUJ in the setting of distal radius malunion. Using in vivo methods, previous authors have documented a reduction in the contact area between the ulnar head and sigmoid notch with malunion. ${ }^{18,19}$ In vivo methods use live subjects with multiplanar distal radius deformities of variable severity. In vitro techniques use cadaveric specimens and allow for individual deformities to be isolated and different conditions to be simulated, such as TFCC rupture. This permits a categorical analysis of the effects of each parameter on the arthrokinematics of the DRUJ.

Accurate indirect measurement of joint contact can be achieved using in vitro techniques. Intercartilage Distance (ICD) is one such technique, which utilizes computed tomography (CT)-based bone and cartilage models, fiducial-based registration, and optical tracking motion capture data. ${ }^{20}$ It has been used previously to characterize DRUJ contact in the intact state. $^{21}$

The purpose of this in vitro study was to utilize ICD to examine the effects of dorsal angulation deformity on DRUJ contact patterns throughout simulated active forearm rotation. Our hypothesis was that the contact area would decrease with progressive dorsal angulation, and that the centroid of contact would become more volar and distal in the sigmoid notch with increasing deformity. We also hypothesized that simulated TFCC rupture would decrease contact area at the sigmoid notch and increase the variability of the contact path of the centroid.

\section{Methods}

\section{Specimen Preparation}

Testing was performed on eight cadaveric forearm specimens (mean age 60 years; range $29-75$ years; six men and two women) with no CT evidence of osteoarthritis. We established that this sample size would provide a power of $80 \%$ to detect changes of $0.8 \mathrm{~mm}$ in centroid position and 20 $\mathrm{mm}^{2}$ in contact area at the 0.05 confidence level. The distal tendons of the wrist extensors (extensor carpi radialis longus [ECRL], extensor carpi ulnaris [ECU]), wrist flexors (flexor carpi radialis [FCR], flexor carpi ulnaris [FCU]), pronator teres [PT], and biceps [BIC] were then sutured using \#2 Ethibond (Ethibond Excel, Ethicon Inc., Piscataway, NJ).

Sutures were passed through alignment guides that reproduced the physiologic line of action of each muscle. ECRL and ECU were routed through a lateral epicondyle sleeve, while PT, FCR, and FCU were routed through a medial epicondyle sleeve. The supinator [SUP] was modeled by placing a suture anchor in the radial tuberosity and routing the attached suture through a Delrin sleeve which traversed the supinator crest to the posterolateral aspect of the ulna. The sutures of ECRL, ECU, FCR, FCU, and SUP were attached to individual pneumatic actuators (Airpot Corporation, Norwalk, CT).

\section{Simulation of Motion}

A servo motor (SM2315D; Animatic, Santa Clara, CA) was used to simulate active motion, with a resistive counterforce provided by a pneumatic actuator. Active supination was initiated by attaching BIC to the servo motor set to motion control at a constant tendon velocity of $5 \mathrm{~mm} / \mathrm{s}$. The muscles were loaded using ratios based on a previous investigation of forearm muscle EMG and cross-sectional area. ${ }^{22}$ Constant tone loads of $10 \mathrm{~N}$ were applied to the FCU, FCR, ECU, and ECRL. Simultaneous pneumatic actuator loads were regulated by proportional pressure controllers (MAC Valves, Wixom, MI) under computer control using custom programmed software (LabVIEW, National Instruments, TX).

\section{Motion Tracking and Kinematic Data Acquisition}

Infrared marker triads were rigidly affixed to the proximal radius and ulna using custom Delrin pedestals and the arc of simulated active supination was tracked using an Optotrak Certus (Northern Digital Inc, Waterloo, Ontario, Canada) optical motion capture system ${ }^{23}$ (-Fig. 1).

\section{Simulation of Distal Radius Deformity}

A previously described, custom-engineered adjustable implant was applied to the volar aspect of the distal radius for each specimen. ${ }^{6}$ This permitted the creation of simulated dorsal angulation deformities. The central appliance of the implant was removable and exchanged for each deformity condition. To install the device, a 20-mm corticocancellous segment of volar distal radius was removed $2 \mathrm{~mm}$ proximal to the DRUJ using an oscillating saw. The dorsal cortex was initially left intact as a bone bridge. Medullary bone from the distal radius metaphysis and shaft was curetted away and cavities were filled with polymethylmethacrylate cement. The adjustable implant was then fixated using bone screws in a neutral position (-Fig. 2 ).

Four different deformity conditions were tested: no deformity (Straight Wedge [SW]), dorsal angulation of 10 degrees (DA10), 20 degrees (DA20), and 30 degrees (DA30). 


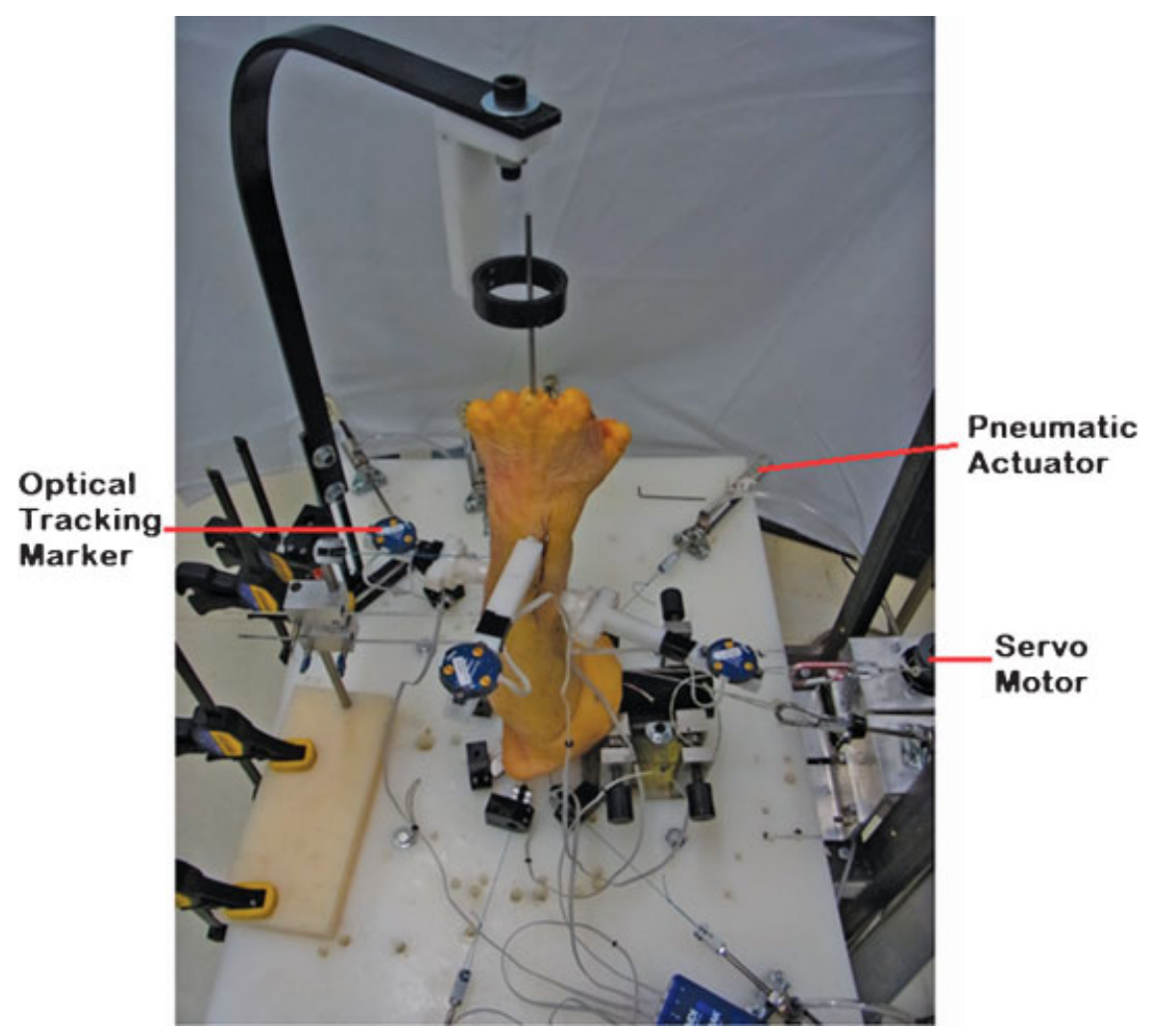

Fig. 1 Depicting a cadaveric specimen mounted in a custom forearm motion simulator. The humerus and ulna are rigidly secured. The outrigger stabilizes a third metacarpal pin holding the radiocarpal joint in a neutral position. Optical tracking markers are mounted on Delrin posts affixed to the radius and ulna. Pneumatic actuators and the servo motor are attached to a Delrin base.

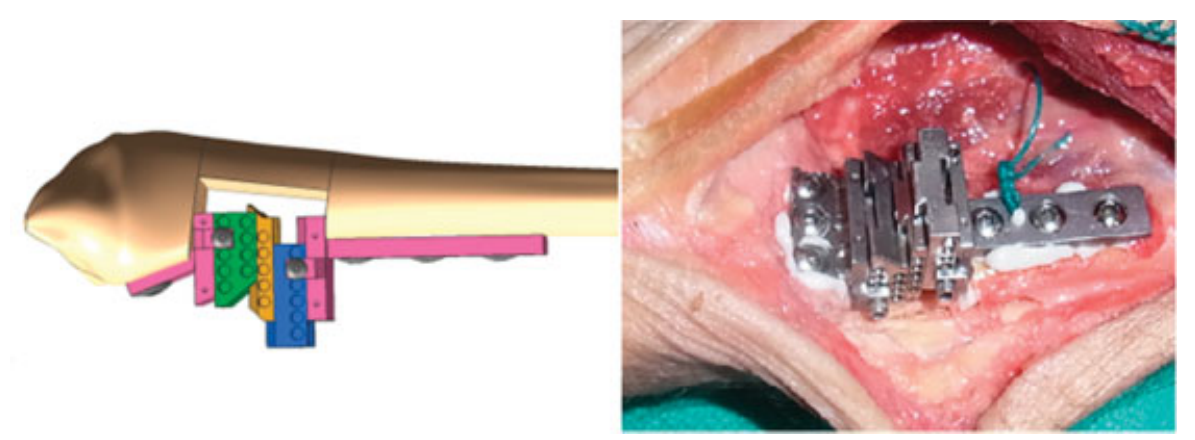

Fig. 2 The custom adjustable implant is inset into the distal radius osteotomy with a dorsal intact bone bridge. Depicted is a schematic and clinical photo, with the implants fixation augmented by intramedullary cement.

The SW configuration of the adjustable implant kept the proximal and distal radius fragments in their original anatomic alignment, while the dorsal angulation configurations introduced progressive dorsal tilt of the articular surface relative to the original anatomy ( - Fig. 3 ).

\section{Testing Procedure}

The specimens were kept hydrated throughout testing using $0.9 \%$ normal saline and closure of the skin envelope between implant exchanges. Kinematic data were gathered with the implant in the neutral (SW) position and for the dorsal angulation deformities with the TFCC intact. Once testing of the intact state had concluded, the TFCC was divided off its ulnar insertion (-Fig. 4).
Subsequently, all deformity testing was repeated for the TFCC insufficient state ( - Fig. 4). At the conclusion of the testing protocol, the forearm was dissected and the bones were denuded of soft tissue. Landmarks on the distal radius, implant, proximal radius, and ulna were digitized relative to the attached motion trackers. This permitted the creation of a three-dimensional anatomic coordinate system; therefore, the kinematic data could be transformed to describe the position of the radius relative to the ulna.

\section{Intercartilage Distance Measurement Technique}

-Fig. 5 provides a flowchart summarizing the stages of data processing which follow kinematic data acquisition to create an ICD measurement. Steps are included from both the 


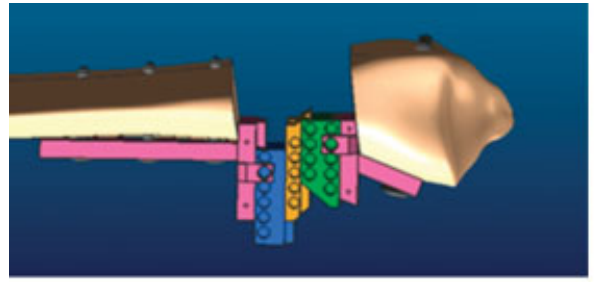

Straight Wedge

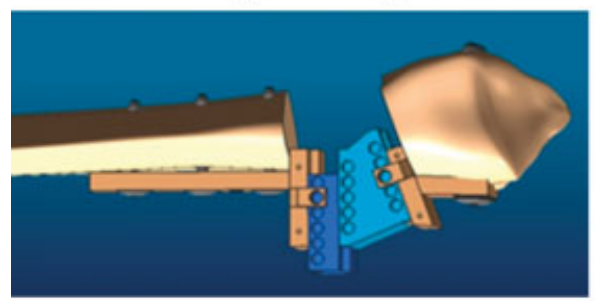

Dorsal Angulation $20^{\circ}$

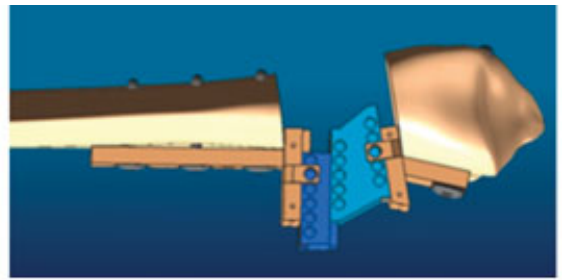

Dorsal Angulation $10^{\circ}$

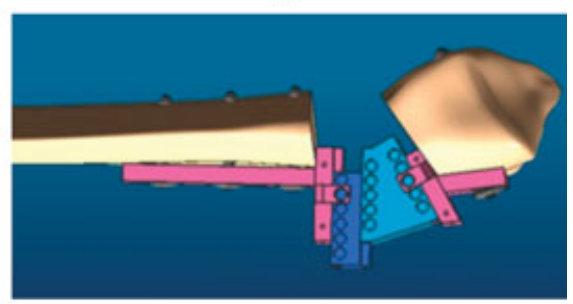

Dorsal Angulation $\mathbf{3 0 ^ { \circ }}$

Fig. 3 Depicting the four different deformity conditions including the straight wedge, dorsal angulation of 10 degrees (DA10), 20 degrees (DA20), and 30 degrees (DA30). Note that the deformities are angulated relative to the original anatomy and do not represent the absolute dorsal angulation value as would be measured on a conventional lateral radiograph.

experimental phase and from the volumetric data acquisition phase using the denuded specimens.

\section{Data Analysis}

All eight specimens were used for ICD contact analysis. The ICD algorithm was used to generate a contact patch and contact centroid for every 10 degrees interval of forearm rotation. The optical tracking system was unable to capture the extremes of forearm rotation due to loss of tracker visualization; therefore, an arc from -60 (60 degrees of supination) to +40 (40 degrees of pronation) was analyzed.

Centroid coordinate data from eight specimens was also evaluated. An anatomical coordinate system was assigned to the sigmoid notch of the distal radius, with a point designated as its center. Contact centroid position relative to the sigmoid notch center was then calculated in $\mathrm{mm}$, for both the proximal-distal $(X)$ and volar-dorsal $(Y)$ axes.

The effects of forearm rotation angle, distal radius deformity, and TFCC sectioning on DRUJ contact area and contact centroid position were evaluated. A three-way repeated measures analysis of variance (ANOVA) was performed, with independent variables of forearm rotation angle, distal radius deformity, and TFCC condition.

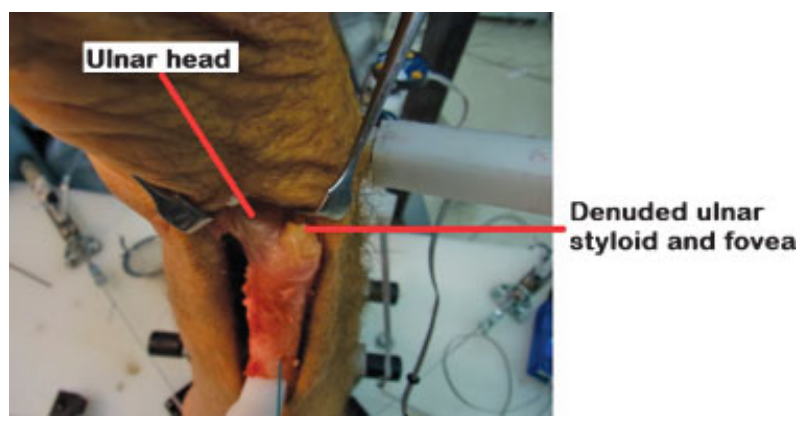

Fig. 4 A photo of the sectioned TFCC, with no residual fibers inserting on the ulnar styloid or fovea. TFCC, triangular fibrocartilage complex.
To determine if the centroid pathways were more variable after TFCC sectioning, the standard deviation values for each 10 degrees interval of forearm rotation were compared using a one-way repeated measures ANOVA for matched deformities. Both the proximal-distal and dorsal-volar axes were assessed.

Data imputation using a linear regression model was used to reconstitute missing contact area and centroid coordinate values. A Greenhouse-Geisser's correction was applied. Statistical significance was set at $p<0.05$. Data presented are the mean DRUJ contact area \pm standard deviation unless otherwise specified. We used a Bonferroni correction for multiple comparisons to compare main effects.

\section{Results}

There was no significant effect from deformity on contact area in the $\operatorname{DRUJ}(p=0.30)$. Forearm rotation angle had a significant effect on contact area $(p=0.004)$, with measurements being highest between 10 and 30 degrees of supination. TFCC sectioning caused a significant decrease in contact area in the DRUJ $(p=0.030)$, with a mean reduction of $11 \pm 7 \mathrm{~mm}^{2}$ between the TFCC intact and sectioned conditions across all variables (-Figs. 6 and 7).

The position of the contact centroid along the volardorsal axis moved volarly with supination for all variables $(p<0.001)$. Deformity had a significant effect on the location of the contact centroid along this plane $(p=0.043)$. Relative to the SW position, the mean centroid position moved $0.3 \pm 1 \mathrm{~mm}$ volar in 10 degrees of dorsal angulation, $0.1 \pm 0.9 \mathrm{~mm}$ volar in 20 degrees of dorsal angulation, and $0.6 \pm 0.9 \mathrm{~mm}$ volar in 30 degrees of dorsal angulation. There was no effect from sectioning the TFCC on the volar-dorsal position of the centroid ( $p=0.24$ ). Variability of the centroid pathway was significantly increased along the volar-dorsal axis after TFCC sectioning ( $p<0.001$ ), with a $16 \%$ increase in the magnitude of standard deviation values for each angle of forearm rotation across deformities. 


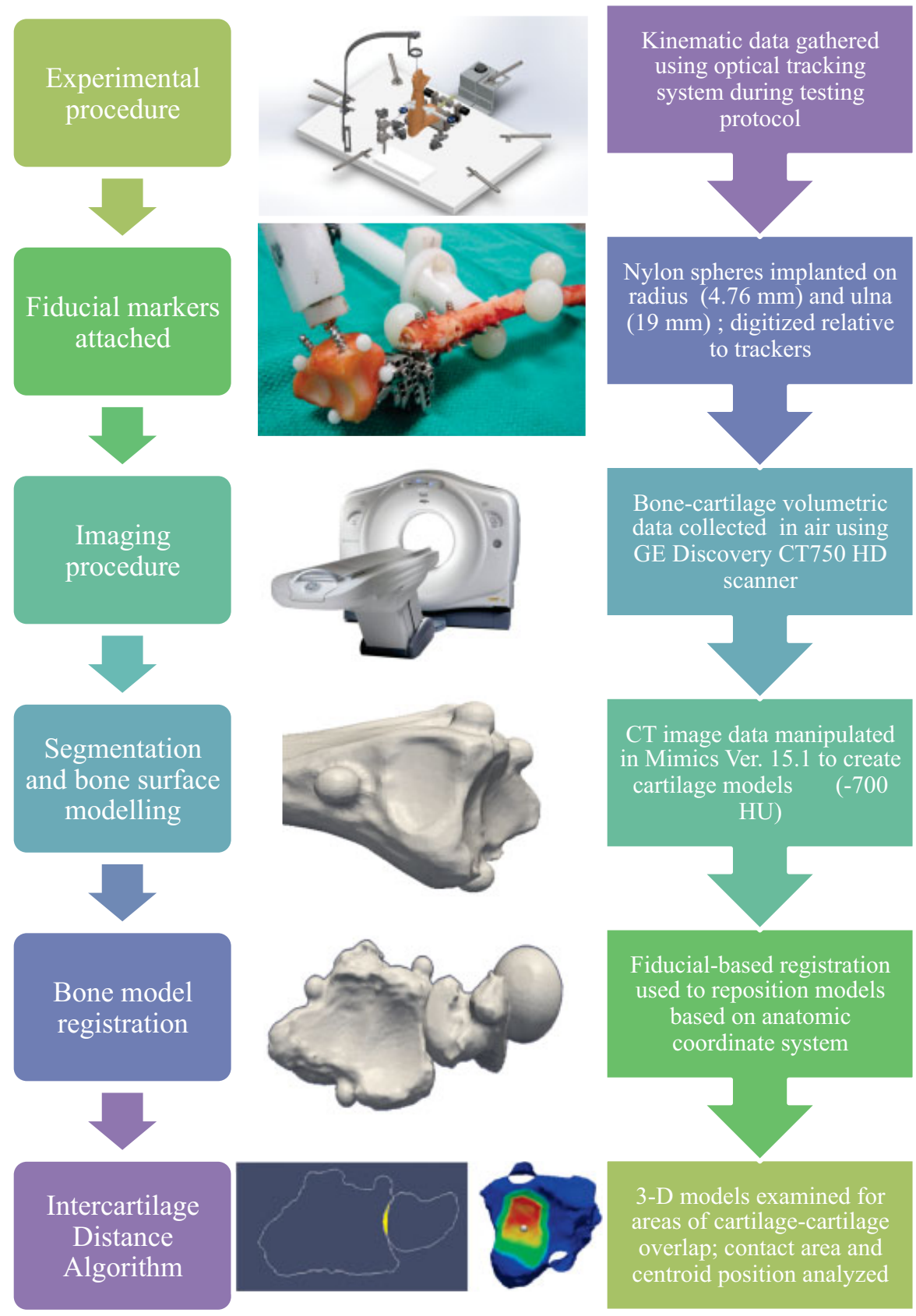

Fig. 5 A flowchart detailing the stages of postexperiment data processing for application of the Intercartilage Distance algorithm.

The position of the contact centroid along the proximaldistal axis moved proximally with supination for all variables $(p=0.043)$. Deformity did not have a significant effect on the location of the contact centroid along this plane $(p=0.17)$. There was no effect from sectioning the TFCC on the proximal-distal position of the centroid $(p=0.21)$. Variability of the centroid pathway was significantly increased along the proximal-distal axis after TFCC sectioning $(p=0.004)$, with a $50 \%$ increase in the magnitude of standard deviation values for each angle of forearm rotation across deformities (-Figs. 8 and $\mathbf{9}$ ).

\section{Discussion}

This study demonstrated that contact area in the DRUJ is variable and dependent on the angle of forearm rotation. Contact area was maximal between 10 and 30 degrees of supination during the conditions tested. These findings are consistent with the literature, with reports indicating that the highest DRUJ contact area values occur across 10 to 30 degrees of supination. ${ }^{24-26}$ We noted that the contact centroid on the sigmoid notch moved volarly and proximally with progressive supination. This was also expected and is in 


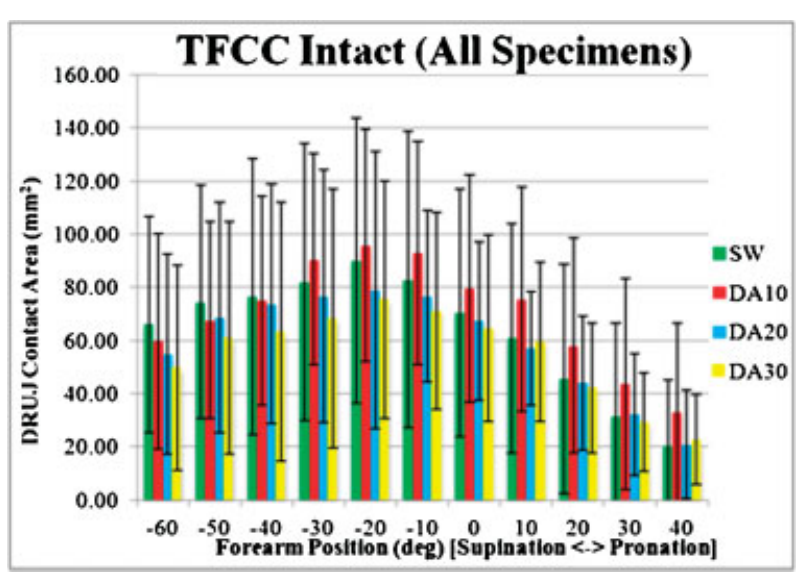

Fig. 6 Depicting the mean +1 SD of DRUJ contact area for the normal condition (SW) and with an increasing degree of dorsal angulation deformity (DA10/20/30). Measurements were made at 10 degrees intervals of forearm rotation, from 60 degrees of supination to 40 degrees of pronation. DRUJ, distal radioulnar joint; SD, standard deviation; SW, straight wedge.

agreement with the published literature on DRUJ kinematics $^{27-30}$ and contact. ${ }^{21,31}$

Simulated malunion with dorsally angulated distal radius deformities influenced DRUJ contact. Increasing dorsal angulation caused the contact centroid to move progressively more volar in the sigmoid notch. This was in keeping with our hypothesis, and relates to the distal radius being dorsally displaced relative to the ulnar head during forearm rotation.,32

We found no correlation between the amount of simulated distal radius deformity and contact area in the DRUJ. This finding was unexpected, given the sensitivity of this technique for subtle contact area changes ${ }^{20}$ and the known effects of dorsal angulation deformity on DRUJ biomechanics. ${ }^{6,7,12,18,32-34}$ It is possible that DRUJ contact area does not change with progressive dorsal angulation of the distal radius. Alternatively, the lack of difference in our study may relate to the arc of motion studied (60 degrees of pronation to 40 degrees of supination). Other authors have noted the greatest effect of deformity at the extremes of forearm rotation, with limitations in pronation ${ }^{3}$

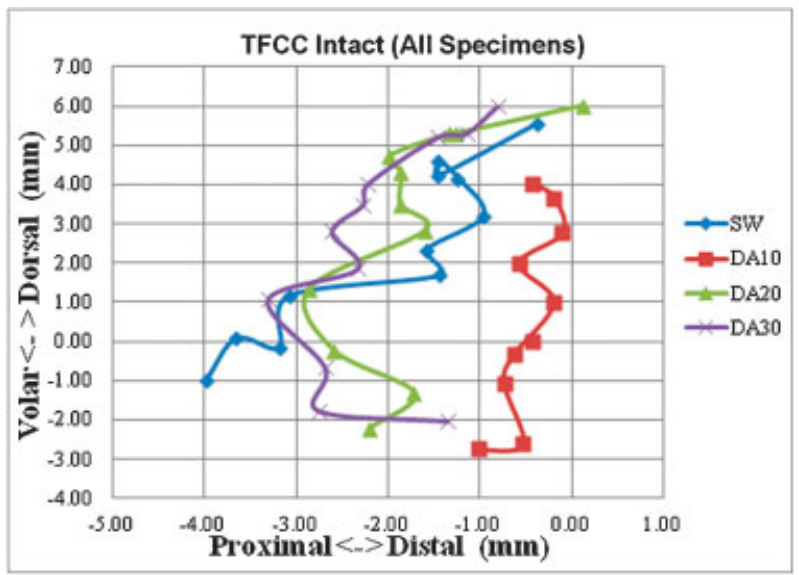

Fig. 8 The position of the contact centroid on the face of the sigmoid notch during forearm rotation. Mean centroid position is displayed for TFCC intact specimens. TFCC, triangular fibrocartilage complex.

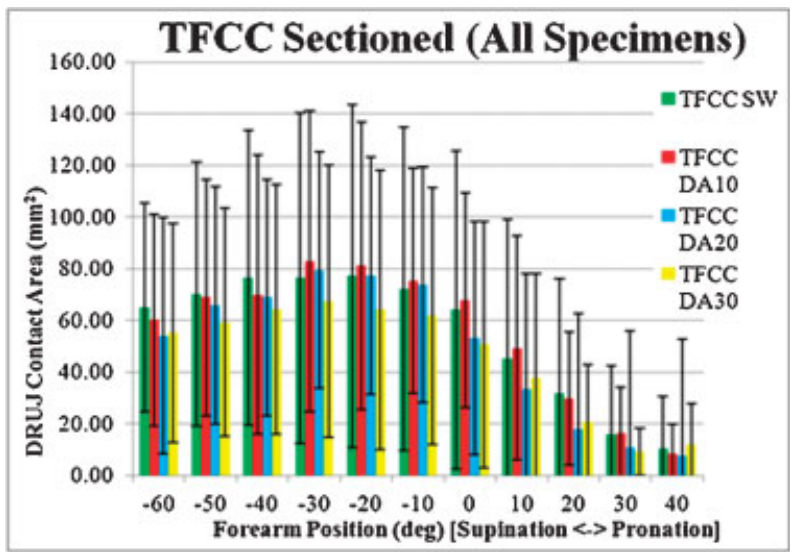

Fig. 7 Depicting the mean +1 SD of DRUJ contact area after TFCC sectioning, for the normal condition (SW) and with an increasing degree of dorsal angulation deformity (DA10/20/30). Measurements were made at 10 degrees intervals of forearm rotation, from 60 degrees of supination to 40 degrees of pronation. DRUJ, distal radioulnar joint; SD, standard deviation; TFCC, triangular fibrocartilage complex.

and supination ${ }^{7}$ beyond 50 degrees of rotation. It is also possible that no difference from deformity was observed because of the type of deformity tested. Previous authors have noted more significant kinematic changes from combined deformities ${ }^{6}$ or shortening compared with dorsal angulation..$^{33}$ Finally, we may have been underpowered with a small sample size to show a statistically significant difference on contact area between deformity groups.

Our findings are interesting to contrast to in vivo studies of the DRUJ in the setting of distal radius malunion. ${ }^{18,34}$ Crisco et a ${ }^{18}$ noted that deformity had a significant effect on interbone joint spacing area (their proxy for joint contact area), but that forearm rotation angle had no effect. They demonstrated less contact in malunited wrists with a contact centroid which moved more proximally. This was in contrast to our findings, which showed a significant effect of forearm rotation angle on contact area, and did not demonstrate a change in DRUJ contact area with deformity. Moreover, unlike Crisco et al, ${ }^{18}$ we noted no change in the position of the contact centroid along the

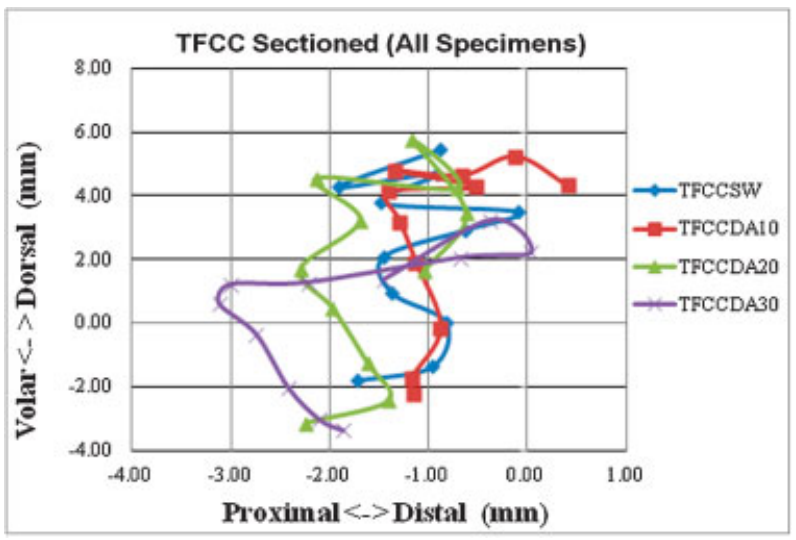

Fig. 9 The position of the contact centroid on the face of the sigmoid notch during forearm rotation. Mean centroid position is displayed for TFCC sectioned specimens. TFCC, triangular fibrocartilage complex. 
proximal-distal axis with deformity, but did find that it displaced volarly with progressive dorsal angulation. Their values for absolute contact area in normal were also significantly higher than in our study and those documented in the DRUJ by other authors using Tekscan. ${ }^{24-26}$ There are multiple reasons that could explain the discrepancies: (1) ICD is more accurate than interbone distance as the true cartilage thickness is accounted for in the bone-cartilage model, compared with interbone distance where an arbitrary number is used to create the proximity map. (2) Their technique uses multiple static positions to extrapolate kinematics; thus, pathways depicting forearm motion may not be entirely accurate. (3) They were evaluating multiplanar deformities which included shortening, as opposed to isolated dorsal angulation deformities as in our study. (4) Their measurements are based on a live population who has an almost complete active range of motion despite their chronic deformity. In vitro specimens are unable to compensate their soft tissue compliance for increasing levels of deformity.

Our study also examined the effect of the TFCC on contact area in the DRUJ. We demonstrated a significant effect of simulated TFCC rupture on contact area in the DRUJ, with a mean contact reduction of $11 \pm 7 \mathrm{~mm}^{2}$ after sectioning. This was to be expected, as once TFCC failure occurs, forces across the DRUJ relax considerably. ${ }^{13}$ It is generally believed that the TFCC complex constrains the DRUJ up to a certain limit in the setting of distal radius deformity. Some authors have experienced that only moderate deformities can be reproduced with an intact TFCC complex..$^{3,35,36}$ Sectioning of the TFCC allows for more extreme malpositions to be achieved ${ }^{6}$ with decreased torques to achieve full prosupination. ${ }^{7}$ In light of the above, it is interesting then that no difference was found from TFCC sectioning on contact centroid position. In our study, after TFCC sectioning, there was a $16 \%$ increase in the magnitude of standard deviation values for the contact centroid position along the dorsal-volar axis, and a 50\% increase along the proximal-distal axis. This implies a dramatic increase in the variability of the contact centroid pathway after sectioning of the TFCC. This variability likely explains why no significant difference was found.

The limitations of this study include the inability for cadaveric specimens to undergo soft tissue adaptation, unlike the in vivo condition. Moreover, the results are less generalizable because only uniplanar deformity was tested, while malunion is usually composed of combination of shortening, angulation, translation, and rotation. The advantage of an in vitro method for studying contact area, compared with in vivo methods, is that test parameters are better controlled and effects of individual deformities can be isolated. Fewer assumptions are made for changes in kinematic pathways, as testing occurs continuously throughout an arc of motion. Finally, the cartilage models created from specimens CT scanned in air create excellent cartilage definition and more accurate models.

In conclusion, increasing dorsal angulation deformity has no apparent effect on contact area in the DRUJ, but causes the contact centroid position to displace volarly. Simulated TFCC rupture reduces the DRUJ contact area and significantly increases the variability of the contact centroid pathway during forearm rotation. Future directions include testing other deformities, including dorsal translation, combined deformities, and volar deformities to increase the generalizability of the results.

\section{Note}

This research was performed in the Hand and Upper Limb Center Biomechanics Laboratory, Lawson Health Research Institute.

\section{Conflict of Interest}

None.

\section{References}

1 Karl JW, Olson PR, Rosenwasser MP. The epidemiology of upper extremity fractures in the United States, 2009. J Orthop Trauma 2015;29(08):e242-e244

2 Bhattacharyya R, Morgan BS, Mukherjee P, Royston S. Distal radial fractures: the significance of the number of instability markers in management and outcome. Iowa Orthop J 2014;34:118-122

3 Kihara H, Palmer AK, Werner FW, Short WH, Fortino MD. The effect of dorsally angulated distal radius fractures on distal radioulnar joint congruency and forearm rotation. J Hand Surg Am 1996;21 (01):40-47

4 Deshmukh SC, Shanahan D, Coulthard D. Distal radioulnar joint incongruity after shortening of the ulna. J Hand Surg $[\mathrm{Br}] 2000 ; 25$ (05):434-438

5 Ishikawa J, Iwasaki N, Minami A. Influence of distal radioulnar joint subluxation on restricted forearm rotation after distal radius fracture. J Hand Surg Am 2005;30(06):1178-1184

6 Fraser GS, Ferreira LM, Johnson JA, King GJ. The effect of multiplanar distal radius fractures on forearm rotation: in vitro biomechanical study. J Hand Surg Am 2009;34(05):838-848

7 Hirahara H, Neale PG, Lin YT, Cooney WP, An KN. Kinematic and torque-related effects of dorsally angulated distal radius fractures and the distal radial ulnar joint. J Hand Surg Am 2003;28(04):614-621

8 Kazuki K, Kusunoki M, Yamada J, Yasuda M, Shimazu A. Cineradiographic study of wrist motion after fracture of the distal radius. J Hand Surg Am 1993;18(01):41-46

9 Tang JB, Ryu J, Omokawa S, Han J, Kish V. Biomechanical evaluation of wrist motor tendons after fractures of the distal radius. J Hand Surg Am 1999;24(01):121-132

10 LaRoque ES, Murray WM, Langley S, Hariri S, Levine BP, Ladd AL. Muscle moment arms in the first dorsal extensor compartment after radial malunion. A cadaver study. J Bone Joint Surg Am 2008; 90(09):1979-1987

11 Kihara H, Short WH, Werner FW, Fortino MD, Palmer AK. The stabilizing mechanism of the distal radioulnar joint during pronation and supination. J Hand Surg Am 1995;20(06):930-936

12 Saito T, Nakamura T, Nagura T, Nishiwaki M, Sato K, Toyama Y. The effects of dorsally angulated distal radius fractures on distal radioulnar joint stability: a biomechanical study. J Hand Surg Eur Vol 2013;38(07):739-745

13 Ferreira LM, Greeley GS, Johnson JA, King GJ. Load transfer at the distal ulna following simulated distal radius fracture malalignment. J Hand Surg Am 2015;40(02):217-223

14 Prommersberger KJ, Pillukat T, Mühldorfer M, van Schoonhoven J. Malunion of the distal radius. Arch Orthop Trauma Surg 2012;132 (05):693-702

15 Brogren E, Wagner P, Petranek M, Atroshi I. Distal radius malunion increases risk of persistent disability 2 years after fracture: a prospective cohort study. Clin Orthop Relat Res 2013;471(05): $1691-1697$ 
16 Cheng HS, Hung LK, Ho PC, Wong J. An analysis of causes and treatment outcome of chronic wrist pain after distal radial fractures. Hand Surg 2008;13(01):1-10

17 Baeyens JP, Van Glabbeek F, Goossens M, Gielen J, Van Roy P, Clarys JP. In vivo 3D arthrokinematics of the proximal and distal radioulnar joints during active pronation and supination. Clin Biomech (Bristol, Avon) 2006;21(Suppl 1):S9-S12

18 Crisco JJ, Moore DC, Marai GE, et al. Effects of distal radius malunion on distal radioulnar joint mechanics-an in vivo study. J Orthop Res 2007;25(04):547-555

19 Xing SG, Chen YR, Xie RG, Tang JB. In vivo contact characteristics of distal radioulnar joint with malunited distal radius during wrist motion. J Hand Surg Am 2015;40(11):2243-2248

20 Willing R, Lapner M, Lalone EA, King GJ, Johnson JA. Development of a computational technique to measure cartilage contact area. J Biomech 2014;47(05):1193-1197

21 Gammon B, Lalone E, Nishiwaki M, Willing R, Johnson J, King GJW. Arthrokinematics of the distal radioulnar joint measured using Intercartilage Distance in an in vitro model. J Hand Surg Am 2018; 43(03):283.e1-283.e9

22 Gordon KD, Dunning CE, Johnson JA, King GJ. Influence of the pronator quadratus and supinator muscle load on DRUJ stability. J Hand Surg Am 2003;28(06):943-950

23 Optotrak Certus. Research-Grade Motion Capture. Manufacturer's Manual. Ontario, Canada: Northern Digital Inc; 2011:1-8

24 Shaaban H, Giakas G, Bolton M, et al. Contact area inside the distal radioulnar joint: effect of axial loading and position of the forearm. Clin Biomech (Bristol, Avon) 2007;22(03):313-318

25 Nishiwaki M, Nakamura T, Nagura T, Toyama Y, Ikegami H. Ulnarshortening effect on distal radioulnar joint pressure: a biomechanical study. J Hand Surg Am 2008;33(02):198-205

26 Malone PS, Cooley J, Morris J, Terenghi G, Lees VC. The biomechanical and functional relationships of the proximal radioulnar joint, distal radioulnar joint, and interosseous ligament. J Hand Surg Eur Vol 2015;40(05):485-493

27 af Ekenstam F, Hagert CG. Anatomical studies on the geometry and stability of the distal radio ulnar joint. Scand J Plast Reconstr Surg 1985;19(01):17-25

28 Schuind F, An KN, Berglund L, et al. The distal radioulnar ligaments: a biomechanical study. J Hand Surg Am 1991;16(06): 1106-1114

29 Linscheid RL. Biomechanics of the distal radioulnar joint. Clin Orthop Relat Res 1992;(275):46-55

30 King GJ, McMurtry RY, Rubenstein JD, Ogston NG. Computerized tomography of the distal radioulnar joint: correlation with ligamentous pathology in a cadaveric model. J Hand Surg Am 1986;11 (05):711-717

31 Chen YR, Tang JB. In vivo gliding and contact characteristics of the sigmoid notch and the ulna in forearm rotation. J Hand Surg Am 2013;38(08):1513-1519

32 Nishiwaki M, Welsh M, Gammon B, Ferreira LM, Johnson JA, King GJ. Distal radioulnar joint kinematics in simulated dorsally angulated distal radius fractures. J Hand Surg Am 2014;39(04): 656-663

33 Adams BD. Effects of radial deformity on distal radioulnar joint mechanics. J Hand Surg Am 1993;18(03):492-498

34 Moore DC, Hogan KA, Crisco JJ III, Akelman E, Dasilva MF, Weiss AP. Three-dimensional in vivo kinematics of the distal radioulnar joint in malunited distal radius fractures. J Hand Surg Am 2002;27 (02):233-242

35 Pogue DJ, Viegas SF, Patterson RM, et al. Effects of distal radius fracture malunion on wrist joint mechanics. J Hand Surg Am 1990;15(05):721-727

36 Scheer JH, Adolfsson LE. Pathomechanisms of ulnar ligament lesions of the wrist in a cadaveric distal radius fracture model. Acta Orthop 2011;82(03):360-364 